\title{
Asymptomatic bacteriuria in type II diabetics and non-diabetics; risk factors, bacterial types and their corresponding resistance pattern
}

\author{
Khalil Y. Abujheisha*(i) \\ Faculty of Pharmacy, Prince Sattam Bin Abdul-Aziz University, Alkharj, Saudi Arabia
}

\section{AR T I C L E IN F O}

Article Type:

Original

\section{Article History:}

Received: 11 September 2019

Accepted: 21 November 2019

Published online: 9 December 2019

Keywords:

Diabetes mellitus

Asymptomatic bacteruria

E. coli

Antibiotics

Urinary tract infection

Extended spectrum beta lactamase

\begin{abstract}
A B S T R A C T
Introduction: Silent bacteriuria or asymptomatic bacteriuria (ASB) means the existence of bacteria in urine without clinical signs or symptoms of the host. Asymptomatic bacteriuria is considered clinically significant and worth treating primarily in pregnant women.

Objectives: In this study, we examined the silent bacteriuria among diabetics and healthy persons, type of strains, and their corresponding resistance pattern.

Materials and Methods: A total of 220 diabetic patients and 70 healthy persons were subjected to study. Diabetic patients without malignancies, asthma or heart diseases, symptoms free regarding urinary tract infection and without prior antibiotic administration were included. Blood samples were obtained from all subjects under aseptic technique for fasting blood sugar and HbAlc. Urine samples were collected. All urine samples were passed to cultivation on suitable culture media. Plates of more than two clinical isolates from the same patient were considered to be contaminated. Plates showing more than $10^{4} \mathrm{CFU} / \mathrm{mL}$ were considered significant ASB and subjected for confirmation of bacterial type and antibiotic susceptibility test.

Results: From diabetics urine samples, 21 (9.55\%) and from healthy persons 3 (4.3\%) showed significant bacterial growth. In both diabetics and non-diabetics, women demonstrated a far higher prevalence of ASB than men. The high the HbAlc, the more possibility of positive ASB. In $66.7 \%$ of ASB, E. coli was the main uropathogens, followed by K. pneumoniae. These species showed identical antibiotic resistance patterns.

Conclusion: These findings emphasize the importance of routine urine culture and antibiotic sensitivity testing for diabetic patients who have identified risk factors. ASB should be treated to avoid potential serious renal complications. Amikacin, gentamicin, piperacillin/tazobactam, fosfomycin, and ciprofloxacin are recommended for treatment of ASB while ampicillin is not.
\end{abstract}

Implication for health policy/practice/research/medical education:

Urinary tract infections are among the most common bacterial infections after upper respiratory tract infections and one of the most common medical complications of pregnancy. Asymptomatic bacteriuria is a type of urinary tract infection which is a common finding in women, diabetics and the elderly. Urinary tract infection causes significant distress to the individual and is associated with high healthcare and social costs. In the United States urinary tract infections are responsible for 7 million clinic visits annually.

Please cite this paper as: Abujheisha KY. Asymptomatic bacteriuria in type II diabetics and non-diabetics; risk factors, bacterial types and their corresponding resistance pattern. J Renal Inj Prev. 2020; 9(1): e02. doi: 10.15171/jrip.2020.02.

\section{Introduction}

Diabetes mellitus (DM) is a metabolic dysfunction marked by hyperglycemia with changes of carbohydrate, protein and fat metabolism as the consequence of deficiencies in either production of insulin, its effectiveness, or both (1). Patients with DM have bladder malfunction which allow urine to accumulate in their pool which serves a suitable environment for microbes to develop and cause infection (2). In the United States, the total estimated cost of diagnosed diabetes was $\$ 245$ billion in 2012, whereas a previous estimate of $\$ 174$ billion in 2007 showed a $41 \%$ increase (3). The major risks for urinary tract infection (UTI) in DM are unsatisfactory glycemic control, the term of DM, recurrent vaginitis, diminished leukocyte function, and anatomical abnormalities and impairment functions of the urinary tract $(2,4,5-7)$.

Silent bacteriuria or asymptomatic bacteriuria (ASB) means the existence of bacteria in urine without clinical 
signs or symptoms of the host. Nevertheless, both diabetic and non-diabetic patients have the possibility of UTIs with or without symptoms $(7,9)$. Diabetic patients are highly susceptible to UTI and up to $35 \%$ of them suffer from UTI $(2,8)$.

Bacteria causing ASB are colonizing flora, which usually arise from the vagina, gut or periurethral area. The most common bacteria that causing ASB isolated from both diabetic patients and non-diabetics are E. coli, Klebsiella spp, Staphylococcus saprophyticus, Staphylococcus aureus, and Candida albicans (9-13).

Asymptomatic bacteriuria is considered clinically significant and worth treating primarily in pregnant women because it has been linked to low-birth weight and preterm birth (14-17). ASB occurs in $2-10 \%$ of women who are pregnant and, where untreated, up to $30 \%$ of the mothers are developing acute pyelonephritis which may cause considerable morbidity if complicated $(14,16,17)$.

This study is designed to investigate ASB among diabetic and nondiabetic patients in AlKharj- Saudi Arabia and to test the etiological agents with the most clinically used antibiotics to facilitate the treatment of the positive cases.

\section{Materials and Methods}

\section{Study population and sample size}

The study carried over diabetic and non-diabetic persons (male and females/age $\geq 18$ ) who came to control blood glucose levels and treat the complications of diabetes during the period of November 2018 - March 2019. A total of 220 diabetic patients were subjected to study in government health care centers in Al-Kharj/Saudi Arabia. In the present study, only diabetic patients without malignancies, asthma or heart diseases, symptoms free regarding UTI and without prior antibiotic administration were considered. Also a total of 70 healthy persons (nondiabetics) have been enrolled as control subjects in the study.

\section{Personal and medical characteristics}

The questionnaire forms were filled after informing the subjects about the aims of the study, participation is voluntary, no risks, personal information will be safe and no financial benefits. The questionnaire includes demographic characteristics of the study population, clinical information about DM patients, diseases, symptoms of UTI (if present) and medication.

\section{Fasting blood sugar}

Blood samples were obtained from subjects study under aseptic technique for fasting blood sugar and HbAlc obtained from the patients' files.

\section{Urine collection and processing}

Before sample collection, every patient had instructions on how to obtain a good midstream urine sample, to prevent sample contamination. Clean voided midstream urine samples $(20 \mathrm{~mL})$ were collected in sterile cups specially for urine collection. All urine samples were passed to cultivation in Microbiology Lab/College of Pharmacy/Prince Sattam University. For isolation of bacteria, $10 \mu \mathrm{L}$ of urine sample was cultivated on suitable culture media such as blood agar, MacConkey agar, and CLED agar (Oxoid, Basingstoke, UK). Inoculated plates were incubated at $37^{\circ} \mathrm{C}$ for $24-48$ hours for visible growth.

\section{Identification of isolated bacteria}

Microbial isolates have been identified following gram staining and standard biochemical tests such as indole, urease, catalase, coagulase, oxidase and citrate. Plates of more than two clinical isolates from the same patient were considered to be contaminated. Plates showing no bacterial growth were considered as sterile. To confirm identifications, positive culture plates were sent to Microbiology Lab/King Khalid hospital.

\section{Susceptibility testing}

Microbiology Lab/King Khalid hospital use Phoenix 100/ BD company machine for identification of bacteria from clinical samples and antibiogram. The antibiotics used for testing gram-negative and gram-positive are given in Tables 3 and 4, and the minimum inhibitory concentration (MIC) results were interpreted according to the Clinical and Laboratory Standard Institute (CLSI) guidelines (18).

\section{Criteria}

Identifying significant ASB in any person is more than $10^{4}$ colony-forming units (CFUs) $/ \mathrm{mL}$ of urine in avoided midstream samples $(4,8,11-13)$.

\section{Ethical issues}

Participation in the study was on voluntary basis. Those willing to participate were requested to sign an informed consent. The study protocol and other study related documents were reviewed and approved by research council of King Fahad Medical City (IRB\# 18-477E).

\section{Statistical analysis}

Data were analyzed using the statistical software SPSS for Windows, version 23.0 (SPSS, IBM). Correlations between variables were calculated with Spearman's rank correlation test. $P<0.05$ was considered to be statistically significant.

\section{Results}

The personal and medical characteristics of DM patients This study was conducted on urine samples from 220 DM type 2 patients, 135 (61.36\%) male and 85 (38.64\%) female in health care centers in Al-Kharj/ Saudi Arabia. In addition, 70 urine samples were collected from healthy persons, 40 (57.1\%) male and 30 (42.9\%) female which were tested for bacterial growth. 
The diabetic patients' mean age was $57 \pm 5$ years old. Based on HbA1c of diabetic subjects, 140 (63.6\%) were having $\leq 8$ while $80(36.4 \%)$ were having more than $8 \%$. The majority of diabetic patients $(135,61.4 \%)$ were not restricted to any diet regimen. Of all diabetics, $70 \%$ have been living with diabetes less than 10 years, the remainder for more than 10 years. Among the diabetic patients, 85 (38.6\%) were suffering from hypertension for which they have used Capoten or amlodipine.

For controlling glucose level, the majority of patients 179 (81.4\%) used metformin only or with either glimepiride or sitagliptin or both. While 41 (18.6\%) of them had either insulin alone or with the previous treatment course. All DM patients had neither signs or symptoms of UTI nor antibiotics. The personal and medical characteristics of DM patients are shown in Table 1.

\section{Significant ASB in diabetics and non-diabetics}

Among the 220 tested diabetics urine samples, 21 (9.55\%) were showing significant bacterial growth with colony count higher than $10^{4} \mathrm{CFU} / \mathrm{mL}$, while 45 (20.45\%) showed mixed growth and the rest of samples $154(70 \%)$ were sterile. Women, 15 out of 85 (17.65\%) showed a higher rate of ASB prevalence than men (6/135, 4.45\%) in the diabetic population. HbAlc $>8(16.3 \%)$ was found to be the significant risk factor for ASB $(P<0.05)$. The distribution of ASB patients according to each risk factor is given in Table 1 .

In healthy persons (non-diabetics), none of the males was positive for ASB while 3/30 (10\%) of females showed

Table 1. The personal and medical characteristics of DM patients

\begin{tabular}{lccc}
\hline Risk factors & Groups & $\begin{array}{c}\text { All diabetics patients } \\
\text { No. (\%) }\end{array}$ & $\begin{array}{c}\text { Positive ASB } \\
\text { No. (\%) }\end{array}$ \\
\hline Gender & Male & $135(61.4)$ & $6(4.4)$ \\
Fge & Female & $85(38.6)$ & $15(17.6)$ \\
HbA1c & $>50$ & $86(39.1)$ & $7(8.14)$ \\
& $>50$ & $134(60.9)$ & $14(10.5)$ \\
DM term/years & $\leq 8$ & $140(63.6)$ & $8(5.7)$ \\
& $>8$ & $80(36.4)$ & $13(16.3)$ \\
Diet & 10 & $154(70)$ & $15(9.7)$ \\
& Yes & $66(30)$ & $6(9.1)$ \\
Hypertension & No & $135(61.4)$ & $8(9.4)$ \\
& Yes & $85(38.6)$ & $9(10.6)$ \\
Treatment & No & $135(61.4)$ & $12(8.9)$ \\
\hline
\end{tabular}

significant growth.

It is worth noting that, the ASB in all diabetics (9.55\%) is more than double compared to healthy persons (4.3\%). The overall information regarding healthy persons is shown in Table 2.

Distribution of bacteria (gram-positive and gramnegative) which were isolated from urine samples of all subjects in the study are exhibited in Figure 1. In both males and females, isolates were clinically pertinent pathogens. As seen in Figure 1, E. coli $(41.7 \%)$ was the leading pathogen isolated followed by $K$. pneumoniae $(25 \%)$

\section{Bacterial susceptibility pattern}

The resistance pattern of all bacteria isolated from diabetic patients and non-diabetics was analyzed and are detailed in Tables 3 and 4 respectively.

S. aureus was identified as MRSA showed a resistance pattern to imipenem, cefoxitin, cefotaxime, ampicillin, penicillin, oxacillin, amoxicillin/clavulanate and sensitive to the rest of the antibiotics. Streptococcus agalactiae appeared resistance to tetracycline only as shown in Table 3.

In fact, the leading pathogen, E. coli, showed relatively high rates of susceptibility for most of the antibiotics tested except for levofloxacin, pipercellin, trimethoprimsulfamethoxazole (80\%) and ampicillin(70\%). It is worth noting that one strain identified as extended spectrum beta-lactamase.

Regarding $K$. pneumoniae, it showed similar patterns of susceptibility compared to $E$. coli but one strain was resistant to trimethoprim-sulfamethoxazole and all of them were resistant to ampicillin. $P$. aeruginosa showed

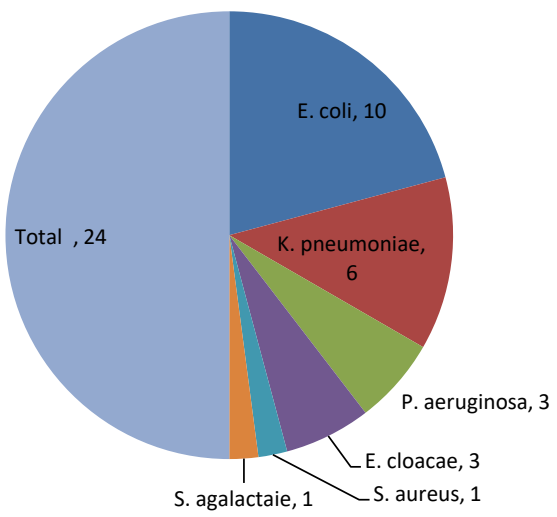

Figure 1. The distribution of bacterial isolates from diabetics and nondiabetics

Table 2. The characteristics of healthy persons

\begin{tabular}{lcccccc}
\hline & Gender & No. & Age & FBS & Diseases & \# Positive ASB \\
\hline \multirow{2}{*}{ Variables } & Male & 40 & $25-65$ & $85-105 \mathrm{mg} / \mathrm{dL}$ & No & None coli, E. cloacae, K. pneumoniae \\
\hline
\end{tabular}


Table 3. Gram-positive bacteria and its sensitivity profile

\begin{tabular}{|c|c|c|}
\hline \multirow[b]{2}{*}{ Antibiotics } & \multicolumn{2}{|c|}{ Bacteria vs. Sensitivity } \\
\hline & $\begin{array}{c}\text { MRSA } \\
\# 1\end{array}$ & $\begin{array}{c}\text { S. agalactiae } \\
\# 1\end{array}$ \\
\hline GN & $S$ & - \\
\hline IMI & $\mathrm{R}$ & - \\
\hline FOX & $\mathrm{R}$ & - \\
\hline CTX & $\mathrm{R}$ & - \\
\hline AMP & $\mathrm{R}$ & $S$ \\
\hline PG & $\mathrm{R}$ & $S$ \\
\hline OX & $\mathrm{R}$ & - \\
\hline AUG & $\mathrm{R}$ & - \\
\hline DAP & S & $\mathrm{s}$ \\
\hline TS & $S$ & - \\
\hline TEIC & $S$ & - \\
\hline VAN & $S$ & $S$ \\
\hline$C D$ & S & $S$ \\
\hline $\mathrm{E}$ & S & - \\
\hline LIN & $S$ & $S$ \\
\hline $\mathrm{MU}$ & $S$ & - \\
\hline $\mathrm{NI}$ & $S$ & - \\
\hline CIP & $S$ & - \\
\hline MOX & $S$ & - \\
\hline RIF & S & S \\
\hline TC & $\mathrm{s}$ & $\mathrm{R}$ \\
\hline
\end{tabular}

Susceptible (S), Resistant (R), No Result (-)

GN: Gentamicin, IMI: Imipenem, FOX: Cefoxitin, CTX: Cefotaxime, AMP: Ampicillin, PG: PenicillinG, OX: Oxacillin, AUG: Amoxicillin/Clavulanate, DAP: Daptomycin, TS: Trimethoprim/Sulfa, TEIC: Teicoplanin, VAN: Vancomycin, CD: Clindamycin, E: Erythromycin, LIN: Linezolid, MU: Mupirocin high level, NI: Nitrofurantoin, CIP: Ciprofloxacin, MOX: Moxifloxacin, RIF: Rifampin, TC: Tetracycline.

sensitive to all tested antibiotics except one species which was resistant to imipenem. Enterobacter cloacae showed resistance to cefuroxime, cefoxitin, cefotaxime, ampicillin, amoxicillin/clavulanate (100\%) and one strain was resistant to tigecycline. The resistance pattern of gram-negative bacteria isolated from urine samples of all subjects in the study are illustrated in Figure 2.

\section{Discussion}

Diabetic patients particularly women have high risk of UTI, we assessed the possible impact of diabetes on the ASB prevalence, bacterial type, and their antibiotic susceptibility pattern. Our key results are; ASB in diabetic patients is much higher than non-diabetics, higher incidence of ASB was recorded in female diabetics and those having $\mathrm{HbA} 1 \mathrm{c}$ more than 8 , since E. coli was the most prevalent etiological agent followed by K. pneumoniae of the total isolated strains which showed good susceptibility pattern with tested antibiotics.

Analysis of the results showed that the risk of ASB is higher among diabetics especially female than their nondiabetic counterparts, in agreement with previous studies $(11,12,19,21,27,28)$. Among the 220 diabetic patients, 9.55\% were positive for bacterial growth which is lesser than studies done in different countries $(20,25,27,28)$,
Table 4. Gram-negative bacteria and its sensitivity pattern

\begin{tabular}{|c|c|c|c|c|}
\hline \multirow[b]{2}{*}{ Antibiotics } & \multicolumn{4}{|c|}{ Bacteria (No.) vs. Sensitivity (\%) } \\
\hline & $\begin{array}{c}\text { E. coli } \\
\# 10\end{array}$ & $\begin{array}{c}\text { K. pneumoniae } \\
\# 6\end{array}$ & $\begin{array}{c}\text { P. aeruginosa } \\
\text { \# } 3\end{array}$ & $\begin{array}{c}\text { E. cloacae } \\
\# 3\end{array}$ \\
\hline AK & 100 & 100 & 100 & 100 \\
\hline GN & 100 & 100 & 100 & 100 \\
\hline ERT & 100 & 100 & 100 & 100 \\
\hline IMI & 100 & 100 & 66.7 & 100 \\
\hline MEM & 100 & 100 & 100 & 100 \\
\hline CXM & 90 & 100 & 100 & 0 \\
\hline FOX & 100 & 100 & 100 & 0 \\
\hline CAZ & 90 & 100 & 100 & 100 \\
\hline CEF & 100 & 100 & 100 & 100 \\
\hline CFX & 100 & 100 & 100 & 0 \\
\hline TOB & 100 & 100 & 100 & 100 \\
\hline CPM & 90 & 100 & 100 & 100 \\
\hline ATM & 90 & 100 & 100 & 100 \\
\hline AMP & 70 & 0 & 100 & 0 \\
\hline AUG & 90 & 100 & 100 & 0 \\
\hline Pip/Taz & 100 & 100 & 100 & 100 \\
\hline PIP & 80 & 100 & 100 & 100 \\
\hline TS & 80 & 83.2 & 100 & 100 \\
\hline FOS & 100 & 100 & 100 & 100 \\
\hline CIP & 100 & 100 & 100 & 100 \\
\hline LEV & 80 & 100 & 100 & 100 \\
\hline TIG & 100 & 100 & 100 & 66.67 \\
\hline
\end{tabular}

AK: Amikacin, GN: Gentamicin, ERT: Ertapenem, IMI: Imipenem, MEM: Meropenem, CXM: Cefuroxime, FOX: Cefoxitin, CAZ: Ceftazidime, CEF: Cefazolin, CFX: Cefotaxime, TOB: Tobramycin, CPM: Cefepime, ATM: Aztreonam, AMP: Ampicillin, AUG: Amoxicillin/Clavulanate, Pip/Taz: Piperacillin/Tazobactam, PIP: Piperacillin, TS: Trimethoprim/Sulfa, FOS: Fosfomycin, CIP: Ciprofloxacin, LEV: Levofloxacin, TIG: Tigecycline.

similar to results obtained by Worku et al, in Ethiopia (22) and higher than those reported in China (3.7\%) by Ke et al (19). This may be attributed because male and female diabetic patients were part of our study whereas former studies included only diabetic women. In the current study, ASB was approximately 4-fold greater in diabetic women than in diabetic males in agreement with previously reported studies $(24,26,29,30)$, which was due to the anatomical properties of female urinary tract. Women have a short and broad urethra close to the anus which facilitate the entrance of bacterial intestines to the urethra. Physiological alteration in the vagina among diabetic women may be another potential cause, including a decrease in normal flora and reduction acidity of vagina $(13,31)$.

In the current study, patient age, duration of DM, insulin treatment and hypertension were not found to be associated with increasing risk for developing ASB. Reports for diabetic patients in Saudi Arabia and other studies have been noted with similar results $(19,27)$. Meanwhile, previous studies reported that age, DM 


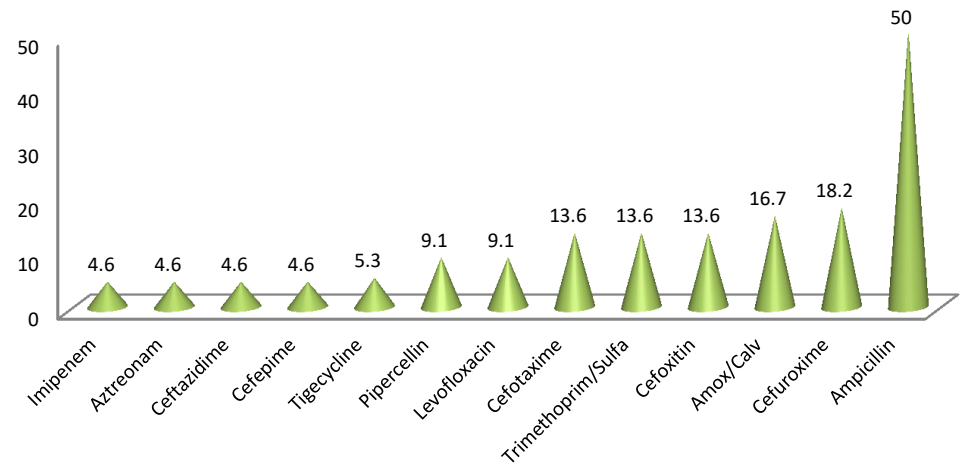

Figure 2. Antibiotics to which gram negative bacteria showed rates of resistance.

term, and hemoglobin A1c levels expose DM patients to symptomatic and asymptomatic UTI $(26,28,29)$. In this study, the only risk factor other than gender for developing ASB was the increasing value of HbAlc. In the previous study in the Saudi diabetic population, Al-Rubeaan et al reported that hypertension and insulin therapy were found to be the significant risk factors for ASB (30).

E. coli $(41.7 \%)$ was the predominant pathogen isolated from urine samples followed by K. pneumoniae (25\%) in agreement of different studies in many countries such as China, Nigeria, Bangladesh, Jordan, Sudan and Romania $(19,23,24,25,27,28)$. While Nabaigwa et al in their study in Uganda found that the most uropathogen isolated was $S$. saprophyticus, followed by E. coli (13).

In this study, the majority of isolated strains showed good susceptibility pattern with the tested antibiotics. The most prevalent pathogens, E. coli and K. pneumoniae, were sensitive to amikacin, gentamicin, imipenem, meropenem, tobramycin, fosfomycin, piperacillin/ tazobactam, ciprofloxacin, and showed resistance to ampicillin, levofloxacin, trimethoprim-sulfamethoxazole, and pipercellin. $P$. aeruginosa showed sensitive to all tested antibiotics except one species which was resistant to imipenem. Enterobacter cloacae was highly resistant to cefuroxime, cefoxitin, cefotaxime, ampicillin, amoxicillin/ clavulanate and sensitive to the rest of tested antibiotics.

Regarding gram-positive bacteria, S. aureus (identified as MRSA) was resistant to imipenem, cefoxitin, cefotaxime, ampicillin, penicillin, oxacillin, amoxicillin/clavulanate and sensitive to gentamicin, trimethoprim/sulfamethoxazole, vancomycin, erythromycin, nitrofurantoin, ciprofloxacin and tetracycline. Streptococcus agalactiae was sensitive to ampicillin, vancomycin, clindamycin, daptomycin, linezolid and resistance to tetracycline only.

Al-Asoufi et al, in a study conducted in Jordan suggested chloramphenicol, ciprofloxacin and vancomycin for treatment of uropathogens and cephalothin was not recommended (25). Hamdan et al nominated cephalexin and gentamicin for treatment but exclude ampicillin, cotrimoxazole, nitrofurantoin, and amoxicillin-clavulanic acid (27). The results obtained by Abdulla et al favored piperacillin/tazobactam, imipenem and amikacin (29) while $\mathrm{Ke}$ et al realized that meropenem and amikacin were the most effective antibiotics on gram-negative bacterial infections but cephalosporins, such as cefoxitin, cefotaxime, cefepime, cefuroxime and cephalothin were not proposed (19).

\section{Conclusion}

ASB is a frequent condition associated with DM patients especially in women. E. coli and K. pneumoniae are the most prevalent etiological agents isolated from urine in diabetics and non-diabetics. It is necessary to do a routine urine analysis, urine culture and antibiotic sensitivity test for diabetic patients who have the identified risk factors. Therefore, ASB should be treated promptly to prevent possible serious renal complications or infections. Amikacin, gentamicin, ertapenem, meropenem, piperacillin/tazobactam, fosfomycin, and ciprofloxacin are recommended for treatment of ASB in DM patients.

Limitations of the study

One of limitations of our study was that we were unable to collect more urine samples because participation in the study was on voluntary basis.

\section{Acknowledgments}

The author wishes to extend his heartfelt appreciation to the King Fahad Medical City/Riyadh for issuing ethical approval and to the patients who agreed to participate in the study. I appreciate the support of the physicians, nursing and technical staff of the health care centers/AlKharj in collecting data and samples. I would like to categorically acknowledge the immense contribution of the staff of Microbiology Laboratory /King Khalid Hospital/ AlKharj for their participation in the confirmation of the isolated bacteria.

\section{Author's contribution}

KYA conceived the study. Handling the samples, performing the experiments, analyzing the data, drafting and revising the final manuscript was also done by the main author. 


\section{Conflicts of interest}

The author declares that he has no conflicts of interest.

\section{Ethical considerations}

Ethical issues (including plagiarism, data fabrication, double publication) have been completely observed by the author.

\section{Funding/Support}

None.

\section{References}

1. Wendy A, Jean B. Clinical Chemistry Text Book. A Laboratory Perspective. 1st ed. USA: F. A. Davis Company. 2007. p. 147-155.

2. Chen SL, Jackson SL, Boyko EJ. Diabetes mellitus and urinary tract infection: epidemiology, pathogenesis and proposed studies in animal models. J Urol. 2009;182:51-6. doi: 10.1016/j.juro.2009.07.090.

3. American Diabetes Association. Economic costs of diabetes in the U.S. in 2012. Diabetes Care. 2013;36:1033-46. doi: $10.2337 / \mathrm{dc} 12-2625$.

4. Geerlings SE. Urinary tract infections in patients with diabetes mellitus: epidemiology, pathogenesis, and treatment. Int J Antimicrob Agents. 2008;31:54-7. Doi: 10.1016/j.ijantimicag.2007.07.042.

5. N Genç Ö, Aksu E, Paşal1-Kilit T, Koçak FE, Korkut Y, Onbaşı K. Asymptomatic bacteriuria, urinary tract infection and risk factors in women with type 2 diabetes mellitus and impaired glucose tolerance. Turk Hij Den Biyol Derg. 2018; 75:365-374. doi: 10.5505/TurkHijyen.2018.59013.

6. Renko M, Tapanainen P, Tossavainen P, Pokka T, Uhari M. Meta-analysis of the significance of asymptomatic bacteriuria in diabetes. Diabetes Care. 2011;34:230-5. doi: $10.2337 / \mathrm{dc} 10-0421$.

7. Boyko EJ, Fihn SD, Scholes D, Chen CL, Normand EH, Yarbro P. Diabetes and the risk of acute urinary tract infection among postmenopausal women. Diabetes Care. 2002;25:1778-83. doi: 10.2337/diacare.25.10.1778.

8. Funfstuck R, Nicolle LE, Hanefeld M, Naber KG. Urinary tract infection in patients with diabetes mellitus. Clin Nephrol. 2012;77:40-8. doi:10.5414/cn107216.

9. Saber M HL, Barai JA, Haq MSA, Jilani MSA, Begum J. The pattern of organism causing urinary tract infection in diabetic and non diabetic patients in Bangladesh. Bangladesh J Med Microbiol. 2010;4:6-8. doi: 10.3329/ bjmm.v4i1.8461.

10. Firas DU. Prevalence of silent bacteriuria in patients with diabetes mellitus. Iraqi Postgraduate Medical Journal. 2008;7: 60-64.

11. Sewify M, Nair SH, Warsame S, Murad M, Alhubail A, Behbehani K, et al. Prevalence of urinary tract infection and antimicrobial susceptibility among diabetic patients with controlled and uncontrolled glycemia in Kuwait. J Diabetes Res. 2016;2016:6573215. doi: 10.1155/2016/6573215.

12. Chukwuocha UM, Emerole CO, Njokuobi TN, Nwawume IC. Urinary tract infections (UTIs) associated with diabetic patients in the Federal Medical Center owerri, Nigeria. Glo Adv Res J Microbiol. 2012;1:62-6.
13. Nabaigwa BI, Mwambi B, Okiria J, Oyet C. Common uropathogens among diabetic patients with urinary tract infection at Jinja Regional Referral Hospital, Uganda. Afr J Lab Med. 2018;7:621. doi: 10.4102/ajlm.v7i1.621.

14. Smaill F, Vazquez JC. Antibiotics for asymptomatic bacteriuria in pregnancy. Cochrane Database Syst Rev. 2007;18:CD000490. doi: 10.1002/14651858.CD000490. pub2.

15. Lin K, Fajardo K. Screening for asymptomatic bacteriuria in adults: evidence for the U.S. Preventive Services Task Force reaffirmation recommendation statement. Ann Intern Med. 2008;149:20-4. doi: 10.7326/0003-4819-149-1200807010-00009-w1.

16. Nicolle LE. Asymptomatic bacteriuria When to screen and when to treat. Infect Dis Clin North Am. 2003;17:367-94.

17. Nicolle LE, Bradley S, Colgan R, Rice JC, Schaeffer A, Hooton TM. Infectious Diseases Society of America; American Society of Nephrology; American Geriatric Society. Infectious Diseases Society of America guidelines for the diagnosis and treatment of asymptomatic bacteriuria in adults. Clin Infect Dis. 2005;40:643-54.doi: $10.1086 / 427507$.

18. Clinical and Laboratory Standards Institute. Performance standards for antimicrobial susceptibility testing; Twenty fourth Informational Supplement M100-24. Wayne, PA, USA: CLSI; 2014.

19. Ke H, Yun Hu, Jun Cheng Shi,Yun Qing Zhu, Xiao Ming Mao. Prevalence, risk factors and microorganisms of urinary tract infections in patients with type 2 diabetes mellitus: a retrospective study in China. Ther Clin Risk Manag. 2018;14:403-8. doi: 10.2147/TCRM.S147078.

20. Alebiosu C, Osinupebi K, Olajubu HJ. Significant asymptomatic bacteriuria among Nigerian type 2 diabetic. Nat Med Assoc. 2003;95:344-351.

21. Kumera B, Anteneh T, Aragaw K. Asymptomatic bacteriuria in relation to diabetic women attending Hawassa University Referral Hospital, Southern Ethiopia. Eur Exp Biol. 2018; 8:5. doi: 10.21767/2248-9215.100046.

22. Worku S, Derbie A, Alemneh Sinishaw M, Adem Y, Biadglegne F. Prevalence of bacteriuria and antimicrobial susceptibility patterns among diabetic and non-diabetic patients attending at Debre Tabor Hospital, Northwest Ethiopia. Int J Microbiol. 2017;2017:5809494. doi: $10.1155 / 2017 / 5809494$.

23. Ifediora A C, Obeagu E I, Chukwudi AI, Priscilla EU. Prevalence of urinary tract infection in diabetic patients attending Umuahia health care facilities. J Bio Innov. 2016: 5:68-82.

24. Shill MC, Huda NH, Moain FB, Karmakar UK. Prevalence of uropathogens in diabetic patients and their corresponding resistance pattern: results of a survey conducted at diagnostic centers in Dhaka, Bangladesh. Oman Med J. 2010;25:282-5. doi: 10.5001/omj.2010.82.

25. Al Asoufi A, Khlaifat A, Tarawneh AA, Alsharafa K, Al Limoun $M$, Khleifat $\mathrm{K}$. Bacterial quality of urinary tract infections in diabetic and non diabetics of the population of Mảan province, Jordan. Pak J Biol Sci. 2017;20:179-88. doi: $10.3923 /$ pjbs.2017.179.188.

26. Wilke T, Boettger B, Berg B, Groth A, Mueller S, Botteman $M$, et al. Epidemiology of urinary tract infections in 
type 2 diabetes mellitus patients: An analysis based on a large sample of 456,586 German T2DM patients. J Diabetes Complications. 2015;29:1015-23. doi: 10.1016/j. jdiacomp.2015.08.021.

27. Hamdan HZ, Kubbara E, Adam AM, Hassan OS, Suliman SO, Adam I. Urinary tract infections and antimicrobial sensitivity among diabetic patients at Khartoum, Sudan. Ann Clin Microbiol Antimicrob. 2015;14:26. doi: 10.1186/ s12941-015-0082-4.

28. Chiţă T, Timar B, Muntean D, Bădițoiu L, Horhat F, Hogea E, et al. Urinary tract infections in Romanian patients with diabetes: Prevalence, etiology, and risk factors. Ther Clin Risk Manag. 2017;13:1-7. doi: 10.2147/TCRM.S123226.
29. Abdulla MC, Jenner F, Alungal J. Urinary tract infection in type 2 diabetic patients: risk factors and antimicrobial pattern. Int J Res Med Sci. 2015;3:2576-9. doi: doi:10.18203/2320-6012.ijrms20150793.

30. Al Rubeaan KA, Moharram O, Al Naqeb D, Hassan A, Rafiullah MR. Prevalence of urinary tract infection and risk factors among Saudi patients with diabetes. World J Urol. 2013;31:573-8. doi: 10.1007/s00345-012-0934-x.

31. Yu S, Fu AZ, Qiu Y, Engel SS, Shankar R, Brodovicz KG, et al. Disease burden of urinary tract infections among type 2 diabetes mellitus patients in the U.S. J Diabetes Complications. 2014;28:621-6. doi: 10.1016/j. jdiacomp.2014.03.012.

Copyright ( $) 2020$ The Author(s); Published by Nickan Research Institute. This is an open-access article distributed under the terms of the Creative Commons Attribution License (http://creativecommons.org/licenses/by/4.0), which permits unrestricted use, distribution, and reproduction in any medium, provided the original work is properly cited. 